\title{
Prognostic Value of Post-diagnosis Health-Related Quality of Life for Overall Survival in Breast Cancer: Findings from a 10-Year Prospective Cohort in Korea
}

\author{
Tran Thi Xuan Mai, MPH ${ }^{1}$ \\ Jin Hyuk Choi, MD² \\ Myung Kyung Lee, RN, $\mathrm{PhD}^{3}$ \\ Yoon Jung Chang, MD, $\mathrm{PhD}^{1,4}$ \\ So-Youn Jung, MD2 \\ Hyunsoon Cho, PhD 1,5 \\ Eun Sook Lee, MD, PhD 2,6
}

\begin{abstract}
${ }^{1}$ Department of Cancer Control and Population Health, National Cancer Center Graduate School of Cancer Science and Policy, Goyang, ${ }^{2}$ Center for Breast Cancer, Research Institute and Hospital, National Cancer Center, Goyang, ${ }^{3}$ College of Nursing, Kyungpook National University, Daegu, ${ }^{4}$ Hospice and Palliative Care Branch, National Cancer Control Institute, National Cancer Center, Goyang, ${ }^{5}$ Division of Cancer Registration and Surveillance, National Cancer Control Institute, National Cancer Center, Goyang, ${ }^{6}$ Department of Cancer Biomedical Science, National Cancer Center Graduate School of Cancer Science and Policy, Goyang, Korea
\end{abstract}

\section{Purpose}

We aimed to evaluate health-related quality of life (HRQOL) at 1-year post-diagnosis in breast cancer $(\mathrm{BC})$ patients and its association with overall survival using data from the National Cancer Center Hospital.

\section{Materials and Methods}

Data of a BC cohort were first obtained between 2004 and 2006 and followed up. HRQOL was assessed using EORTC QLQ-C30 and BC specific module QLQ-BR23 few days after diagnosis and 1 year after that. We examined and compared the difference in the two HRQOL scores measured for each patient by the patient's current survival status. The Cox proportional hazards model was fitted to evaluate the impact of HRQOL on survival, with adjustment for baseline HRQOL and other factors.

\section{Results}

Of 299 enrolled patients, 206 responded at 1-year post-diagnosis (80.6\%) and were followed up for 11.6 years on average. At 1-year post-diagnosis, survivors had better HRQOL scores than those who died, although their health status was similar at baseline. Survivors reported significant increase 1 year after diagnosis in global health status and emotional scales. Between the groups, functional scales such as physical, role, and emotional were significantly different. Functional scales, including physical (adjusted hazard ratio [aHR], 0.70 ), role (aHR, 0.68), emotional (aHR, 0.72), and symptom scales, including fatigue (aHR, 1.34), dyspnea (aHR, 1.29), appetite loss (aHR, 1.24) were significantly associated with overall survival. Patients who were less worried about future health had favorable survival (aHR, 0.83).

\section{Conclusion}

Besides treatment-related symptoms, non-medical aspects at 1-year post-diagnosis, including functional well-being and future perspective, are predictive of long-term survival. Intervention to enhance physical, role, and emotional support for women soon after their BC diagnosis might help to improve disease survival outcomes afterwards.

\section{Key words}

Breast neoplasms, Quality of life, Overall survival 


\section{Introduction}

It has become increasingly accepted that self-reports from cancer patients provide exclusive perspectives and address aspects of wellbeing, feelings, and functioning which may not otherwise be captured by standard clinical assessments [1]. Therefore, patient-reported outcome, specifically healthrelated quality of life (HRQOL), is considered an important outcome measure in cancer clinical trials [2] and shows consistency and reliability in association with clinical outcomes in both oncology and non-oncology fields [3,4]. Assessing quality of life (QOL) in cancer patients could improve treatment and be used as a medical prognostic factor for survival [5-8]. Results from QOL studies can further recommend the direction needed for more efficient treatment for cancer patients [9].

Previous studies measuring HRQOL focused on comparing patients' HRQOL between different treatments or measuring the longitudinal impact of treatment on HRQOL at different treatment phases. However, interests have shifted to finding the relationship between HRQOL and survival since data in the literature show that HRQOL predicts survival in different types of cancers independent of other clinical factors $[2,10,11]$. The number of studies reporting the relationship between HRQOL and survival of breast cancer (BC) patients is limited [12]. The most common study design was by randomized controlled trials [9,11,13-17]; two studies followed a cohort design [18,19], and two were systematic reviews and meta-analyses $[1,20]$. These studies utilized the baseline QOL, which was usually measured a few days to two weeks before treatment, and its association with survival of BC patients. Results from these studies, however, were inconsistent. Some reported that baseline QOL provided useful prognostic information [13,19]; others, conversely, reported that baseline QOL had no great importance in predicting primary clinical endpoints, including overall survival (OS) $[11,16]$. Other studies reported that QOL scores predicted outcome in metastatic [21] but not in early BC [14].

The relationship between HRQOL outcomes, symptoms, and long-term survival have not been completely elucidated [9]. From previous studies, baseline QOL might not be a prognostic factor, especially for non-metastatic BC patients. Due to the inconsistency of previous studies, we shifted our interest to the QOL measured one year after patients were diagnosed, with the hypothesis that the patients' QOL status at this time point has better prognostic value for OS. Using the BC cancer cohort data at the National Cancer Center (NCC) of Korea, we evaluated the association between 1-year post-diagnosis HRQOL and OS, with adjustment for QOL status at baseline.

Thus, we explored which dimensions of HRQOL after pati- ents finished treatment would be useful to predict their survival. To our knowledge, this is one of the few studies evaluating the prognostic significance of HRQOL measured at this specific time point with a long-term follow-up period. Our results might further evidence on utilizing HRQOL as a survival prognostic factor.

\section{Materials and Methods}

\section{Study design and subject recruitment}

In May 2004, a BC prospective cohort was set up to collect data on issues of cancer survivorship at the NCC in Korea. Among 371 patients diagnosed between May 2004 and September 2006, 299 agreed to participate in our cohort study (Fig. 1). Eligibility criteria were histologically confirmed invasive cancer (stage I-IV), being 18 years or older, and absence of other cancers. Participants were prospectively followed up according to the study protocol. More details on patients' recruitments elsewhere [22,23].

\section{Data collection}

$\mathrm{BC}$ patients were diagnosed by oncologists at the Breast Cancer Department of NCC hospital. We explained the purpose and protocol of the study to all participants. The survey was based on self-reported data. The first data collection was conducted a few days after diagnosis. After the interview, subjects were given a baseline questionnaire together with a stamped and addressed return envelope. One year after diagnosis, each participant was sent a letter with a follow-up questionnaire, a stamped and addressed return envelope. Those who did not return the questionnaire within 2 weeks were contacted by phone up to three times. Data on survival were obtained through linkage with the national death registration database, with the last data collection done in July 2018.

\section{Measures}

\section{1) Sociodemographic and clinical characteristics}

Age at diagnosis and level of education were measured one time at baseline. Other sociodemographic characteristics, including religious beliefs, job status, monthly household income, and marital status were measured at both baseline and 1-year post-diagnosis. Data on comorbidity status and health behaviors (cigarette smoking and alcohol drinking) were also collected at both measures. In the current study, 


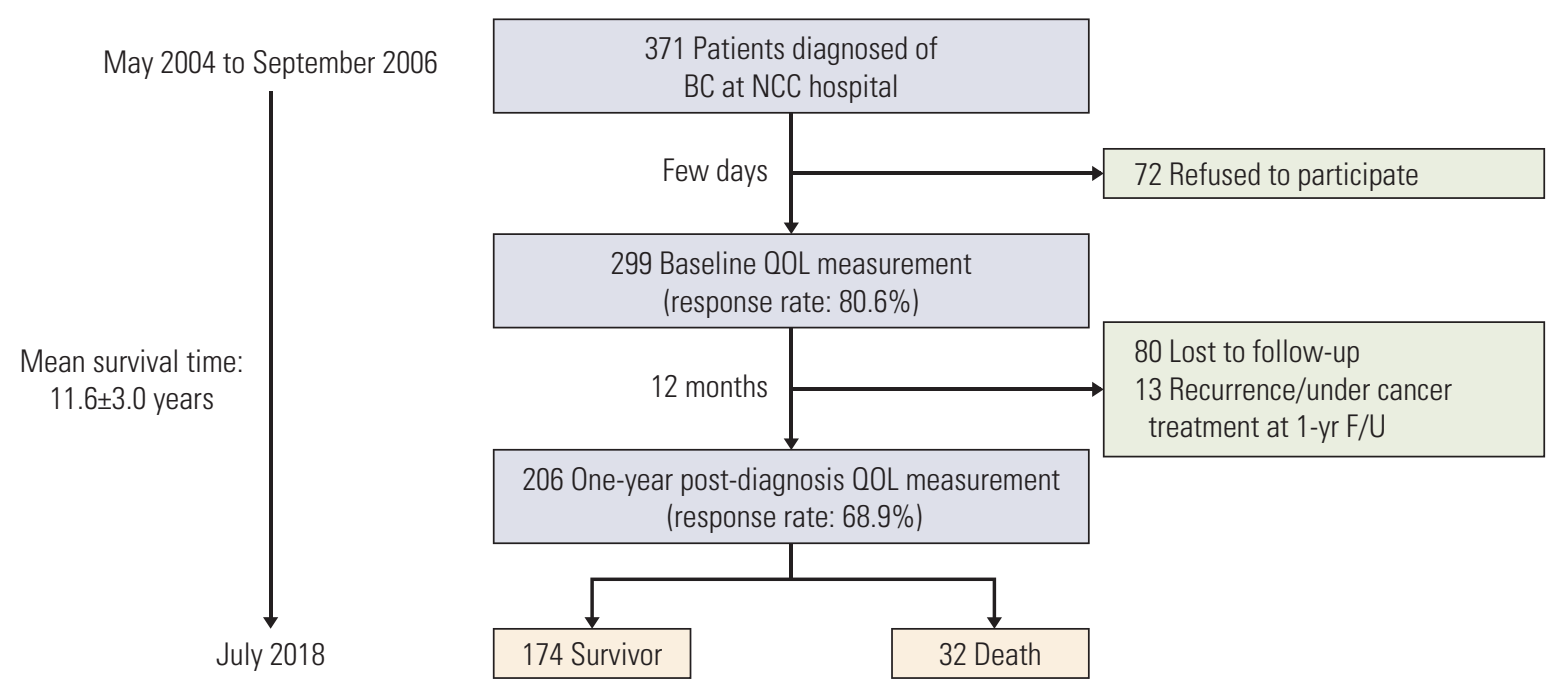

Fig. 1. Enrollment of cohort, health-related quality of life (QOL) assessments and follow-up period. BC, breast cancer; NCC, National Cancer Center; F/U, follow-up.

we presented the results measured at 1-year post-diagnosis for these variables. Medical information obtained from the NCC hospital electronic medical records included stage at diagnosis, radiotherapy (yes or no), duration of chemotherapy (days), chemotherapy (yes or no), duration of chemotherapy (days), hormone therapy (yes or no), and BC subtype. Stage at diagnosis was categorized into two groups: stage I to IIA and stage IIB to IV. BC subtype variable has four values: estrogen receptor (ER)/ progesterone receptor (PR) positive and human epidermal growth factor receptor (HER2) positive; ER/PR positive and HER2 negative; ER/PR negative and HER2 positive; ER/PR negative and HER2 negative.

\section{2) Health-related quality of life}

HRQOL was assessed using the European Organization for Research and Treatment of Cancer (EORTC) general module QLQ-C30 [24] and BC module QLQ-BR23 [25]. These are well-validated and commonly utilized instruments in the oncology field $[2,26]$. EORTC QLQ-C30 assesses a patient's capacity to fulfill the activities of daily living, comprising of five functional scales, six single symptom items, and a global health status scale. The QLQ BR-23 module incorporates multi-item and single item scales to assess systemic therapy side effects, breast symptoms, and other BC QOL scales. In both instruments, higher functional scores and higher global health status represent better functioning and QOL whereas a higher symptom score indicates more severe symptoms or worse QOL. The Korean versions of both instruments were validated in previous research. Cronbach's alpha was greater than 0.7 for the Korean validated version of both instruments $[27,28]$. HRQOL assessments were performed at two-time points: immediately after diagnosis (baseline) and 1-year post-diagnosis.

\section{3) Data analysis and statistical methods}

Demographic characteristics and clinical factors were summarized using frequencies and percentages for categorical variables, and means and their standard deviations or medians and their interquartile ranges for continuous variables, with stratification by survival status. Statistical significance of differences between groups in categorical variables were tested by Fisher exact test. The normality of the distribution of continuous variables was tested by the Shapiro-Wilk test. Statistical significance of differences between groups in continuous variables was tested by the Student's $t$ test for normally distributed variables and the Kruskal-Wallis test for non-normally distributed variables. Statistical significance of differences in QOL scores between baseline and 1-year postdiagnosis was tested by non-parametric Wilco-xon signedrank test.

Patient survival was defined as the time interval between the date of $\mathrm{BC}$ diagnosis to the date of death from any cause or the date of the last follow-up. Demographic, clinical and QOL variables were evaluated using univariate Cox proportional hazards models. In all multivariate models, age at diagnosis, stage at diagnosis, and baseline HRQOL were adjusted. For other factors, backward model selection was performed to choose variables to be included in the multivariate model. For each HRQOL scale from QLQ-C30 and 
Table 1. Demographic and clinical characteristics of the study population

\begin{tabular}{|c|c|c|c|c|}
\hline One-year post-diagnosis & Total $(\mathrm{n}=206)$ & Survivor $(\mathrm{n}=174)$ & Death $(n=32)$ & p-value \\
\hline \multicolumn{5}{|l|}{ Demographic variable } \\
\hline \multicolumn{5}{|l|}{ Age at diagnosis (yr) } \\
\hline $20-44$ & $71(34.5)$ & $60(34.5)$ & $11(34.4)$ & 0.321 \\
\hline $45-54$ & $78(37.9)$ & $69(39.7)$ & $9(28.1)$ & \\
\hline$\geq 55$ & $57(27.7)$ & $45(25.9)$ & $12(37.5)$ & \\
\hline Mean \pm SD & $47.3 \pm 9.1$ & $47.0 \pm 9.1$ & $48.7(9.7)$ & $0.353^{\mathrm{a})}$ \\
\hline \multicolumn{5}{|c|}{ Education level at diagnosis } \\
\hline$\leq$ High school & $151(73.7)$ & $126(72.4)$ & $25(80.7)$ & 0.338 \\
\hline z University & $54(26.3)$ & $48(27.6)$ & $6(19.3)$ & \\
\hline \multicolumn{5}{|l|}{ Having religion } \\
\hline No & $52(25.5)$ & $48(27.9)$ & $4(12.5)$ & 0.066 \\
\hline Yes & $152(74.5)$ & $124(72.1)$ & $28(87.5)$ & \\
\hline \multicolumn{5}{|l|}{ Job status } \\
\hline No & $117(62.6)$ & $99(63.5)$ & $18(58.1)$ & 0.571 \\
\hline Yes & $70(37.4)$ & $57(36.5)$ & $13(41.9)$ & \\
\hline \multicolumn{5}{|l|}{ Monthly income (USD) } \\
\hline$<2,000$ & $81(43.1)$ & $68(42.0)$ & $13(50.0)$ & 0.443 \\
\hline$\geq 2,000$ & $107(56.9)$ & $94(58.0)$ & $13(50.0)$ & \\
\hline \multicolumn{5}{|l|}{ Marital status } \\
\hline Married & $166(81.0)$ & $139(80.4)$ & $27(84.4)$ & 0.594 \\
\hline Others & $39(19.0)$ & $34(19.7)$ & $5(15.6)$ & \\
\hline \multicolumn{5}{|l|}{ Drinking } \\
\hline Yes & $16(8.3)$ & $147(90.7)$ & $29(96.7)$ & 0.281 \\
\hline No & $176(91.7)$ & $15(9.3)$ & $1(3.3)$ & \\
\hline \multicolumn{5}{|l|}{ Smoking } \\
\hline Yes & $3(1.6)$ & $161(98.8)$ & $28(96.6)$ & 0.374 \\
\hline No & $189(98.4)$ & $2(1.2)$ & $1(3.5)$ & \\
\hline \multicolumn{5}{|l|}{ Clinical characteristic } \\
\hline \multicolumn{5}{|l|}{ Comorbidity status } \\
\hline No & $130(69.2)$ & $116(73.0)$ & $14(48.3)$ & 0.008 \\
\hline Yes & $58(30.9)$ & $43(27.0)$ & $15(51.7)$ & \\
\hline \multicolumn{5}{|l|}{ Radiotherapy } \\
\hline No & $43(21.0)$ & $36(20.8)$ & $7(21.9)$ & 0.892 \\
\hline Yes & $162(79.0)$ & $137(79.2)$ & $25(78.1)$ & \\
\hline \multicolumn{5}{|c|}{ Duration of radiotherapy (day) } \\
\hline Mean \pm SD & $46.7 \pm 21.5$ & $45.5 \pm 11.2$ & $53.8 \pm 51.0$ & $0.093^{\mathrm{a})}$ \\
\hline \multicolumn{5}{|l|}{ Chemotherapy $^{\mathrm{b})}$} \\
\hline No & $28(13.7)$ & $28(16.2)$ & 0 & NA \\
\hline Yes & $177(86.3)$ & $145(83.8)$ & $32(100)$ & \\
\hline \multicolumn{5}{|c|}{ Duration of chemotherapy (day) } \\
\hline Mean \pm SD & $116.0 \pm 42.8$ & $113.8 \pm 36.3$ & $125.6 \pm 64.6$ & $0.161^{\text {a) }}$ \\
\hline \multicolumn{5}{|l|}{ Hormone therapy } \\
\hline No & $39(18.9)$ & $35(20.1)$ & $4(12.5)$ & 0.312 \\
\hline Yes & $167(81.1)$ & $139(79.9)$ & $28(87.5)$ & \\
\hline \multicolumn{5}{|l|}{ Stage } \\
\hline I-IIA & $131(63.6)$ & $121(69.5)$ & $10(31.3)$ & $<0.001$ \\
\hline IIB-IV $\left.{ }^{c}\right)$ & $75(36.4)$ & $53(30.5)$ & $22(68.8)$ & \\
\hline
\end{tabular}

(Continued to the next page) 
Table 1. Continued

\begin{tabular}{|c|c|c|c|c|}
\hline One-year post-diagnosis & Total $(n=206)$ & Survivor $(\mathrm{n}=174)$ & Death $(n=32)$ & p-value \\
\hline \multicolumn{5}{|c|}{ Breast cancer tumor subtypes ${ }^{\mathrm{b})}$} \\
\hline ER/PR+, HER2+ & $11(5.4)$ & $10(5.8)$ & $1(3.2)$ & NA \\
\hline ER/PR+, HER2- & $156(76.1)$ & $129(74.1)$ & $27(87.1)$ & \\
\hline ER/PR-, HER2+ & $13(6.3)$ & $11(6.3)$ & $2(6.5)$ & \\
\hline ER/PR-, HER2- & $25(12.2)$ & $24(13.8)$ & $1(3.2)$ & \\
\hline
\end{tabular}

Values are presented as number $(\%)$ unless otherwise indicated. SD, standard deviation; ER, estrogen receptor; PR, progesterone receptor; HER2, human epidermal growth factor receptor 2. ${ }^{a}$ p-value was obtained from the Kruskal-Wallis test, b)NA, not available (due to small number of participant), ')Five participants with stage IV. Other p-values were obtained by Fisher's exact test.

QLQ-BR23, we fitted a Cox model; with survival status as the response variable, the main predictor variable was HRQOL score.

For HRQOL variables, the raw scores were linearly transformed to give standard scores in the range of 0-100 for each of the functional and symptom scales according to the EORTC scoring manual [29]. A difference of 5-10 points in QOL scores represents a small change, 10-20 points a moderate change and greater than 20 points a large, clinically significant change from the patient's perspective. In the current study, we presented hazard ratios (HR) for a 10-point change on the continuous HRQOL variables [20,30]. All tests were two-sided; p-values less than 0.05 were considered statistically significant. Statistical analyses were conducted using SAS statistical software ver. 9.2 (SAS Institute Inc., Cary, NC) and figures were visualized in R Studio Software R ver. 3.5.1, 2018 (The R Foundation for Statistical Computing Platform, Vienna, Austria).

\section{Ethical statement}

The protocol and follow-up of this study were approved by the NCC Institutional Review Board (NCCNCS-04-034). All patients provided written informed consent prior to participation.

\section{Results}

\section{Patient characteristics}

Among 371 women diagnosed with BC at the NCC, 299 agreed to participate in our study $(80.6 \%)$ and their baseline HRQOL was measured. Of these, 206 patients replied at 1-year post-diagnosis. Of the 206 patients, 32 deaths were reported as at July 2018. The follow-up period was 11.6 years on average with a 5-year OS rate of $93.2 \%$ and a 10 -year rate of $87.7 \%$ (Fig. 1). Table 1 describes demographic and clinical characteristics, classified according to survival status. Mean age was $47.3 \pm 9.1$ at diagnosis, $79.0 \%$ had radiotherapy and $86.3 \%$ had chemotherapy. Notably, all deaths in our cohort had chemotherapy. Comorbidity status and stage at diagnosis were significantly different between the survivor and death groups. In death group, 51.7\% had at least one comorbidity versus $30.9 \%$ in survivor groups. In addition, in death group, $68.8 \%$ were diagnosed at stage IIB to IV compared to $30.5 \%$ in survivor group. These features in the survivor group were $27.0 \%$ and $30.5 \%$ respectively.

\section{HRQOL at baseline and 1-year post-diagnosis}

Table 2 displays HRQOL scores at baseline and 1-year post-diagnosis by survival status for all scales of QOL-C30 and QLQ-BR23. The global health status mean score of the

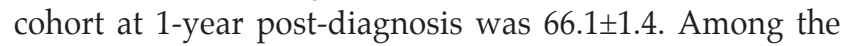
functional scales, at 1-year post-diagnosis the social scale had the lowest mean score $(71.1 \pm 1.9)$ while the physical scale had the highest mean score $(76.0 \pm 1.1)$. In both the survivor and death groups, QOL deteriorated at 1-year post-diagnosis compared to baseline, with an increase in the symptom scales and decrease in the functional scales. However, compared to the survivor group, the differences in the death group were greater in several scales. For example, the difference was 6.2 points $(p<0.001)$ in the physical scale of the survivor group while it was 15.5 points $(\mathrm{p}<0.001)$ in the death group.

Figs. 2, 3, and 4 illustrate the trend of HRQOL, measured by EORTC QLQ-C30, and QLQ-BR23 respectively, from baseline to 1-year post-diagnosis in survivors and deaths. At baseline, QOL scores of both survivors and deaths were similar while at 1-year post-diagnosis, there existed larger differences between these two groups. The survivor group had a significantly better QOL at 1-year post-diagnosis in most 
Table 2. HRQOL mean scores (QLQ-C30 and QLQ-BR23) at baseline and 1-year post-diagnosis

\begin{tabular}{|c|c|c|c|c|c|c|c|c|c|c|c|}
\hline \multirow{2}{*}{ QOL scale } & \multirow{2}{*}{$\begin{array}{c}\text { No. } \\
(n=206)\end{array}$} & \multicolumn{2}{|c|}{ Total at $1 \mathrm{yr}$} & \multicolumn{4}{|c|}{ Survivor } & \multicolumn{4}{|c|}{ Death } \\
\hline & & Mean & SD & Base & $1 \mathrm{yr}$ & Diff. & p-value ${ }^{a)}$ & Base & $1 \mathrm{yr}$ & Diff. & p-value ${ }^{a)}$ \\
\hline Global health status & 206 & 66.1 & 1.4 & 55.2 & 67.4 & 12.2 & $<0.001$ & 61.0 & 59.4 & -1.6 & 0.906 \\
\hline \multicolumn{12}{|l|}{$\begin{array}{c}\text { EORTC QLQ-C30 } \\
\text { Functional Scale }\end{array}$} \\
\hline Physical & 206 & 76.0 & 1.1 & 83.7 & 77.6 & -6.2 & $<0.001$ & 82.9 & 67.4 & -15.5 & $<0.001$ \\
\hline Role & 206 & 73.7 & 1.6 & 85.9 & 75.9 & -10.0 & $<0.001$ & 81.3 & 62.0 & -19.3 & 0.001 \\
\hline Emotional & 205 & 73.3 & 1.4 & 63.3 & 74.9 & 11.6 & $<0.001$ & 63.7 & 64.7 & 1.0 & 0.511 \\
\hline Cognitive & 205 & 74.3 & 1.3 & 81.7 & 75.0 & -6.7 & $<0.001$ & 82.3 & 70.3 & -12.0 & 0.012 \\
\hline Social & 201 & 71.1 & 1.9 & 75.1 & 72.6 & -2.5 & $<0.001$ & 74.7 & 63.0 & -11.7 & 0.089 \\
\hline \multicolumn{12}{|l|}{ EORTC QLQ-C30 } \\
\hline Fatigue & 205 & 36.6 & 1.5 & 32.5 & 34.5 & 2.0 & 0.141 & 32.6 & 48.1 & 15.5 & 0.015 \\
\hline Nausea and vomiting & 205 & 7.1 & 0.9 & 7.2 & 6.4 & -0.8 & 0.509 & 8.6 & 10.9 & 2.3 & 0.247 \\
\hline Pain & 206 & 22.9 & 1.5 & 14.0 & 21.4 & 7.4 & $<0.001$ & 16.7 & 31.3 & 14.6 & 0.042 \\
\hline Dyspnea & 205 & 16.7 & 1.6 & 13.7 & 14.7 & 1.0 & 0.571 & 13.5 & 27.1 & 13.5 & 0.029 \\
\hline Insomnia & 205 & 23.0 & 1.8 & 20.8 & 21.8 & 1.0 & 0.672 & 22.9 & 29.2 & 6.3 & 0.240 \\
\hline Appetite loss & 205 & 15.5 & 1.7 & 19.1 & 14.7 & -4.3 & 0.047 & 18.8 & 19.8 & 1.0 & 0.901 \\
\hline Constipation & 204 & 18.3 & 1.7 & 14.8 & 16.5 & 1.6 & 0.327 & 22.6 & 28.1 & 5.5 & 0.379 \\
\hline Diarrhea & 205 & 8.0 & 1.1 & 10.2 & 7.3 & -2.9 & 0.046 & 10.4 & 11.5 & 1.0 & 0.948 \\
\hline Financial difficulties & 200 & 28.0 & 2.0 & 23.9 & 25.8 & 1.9 & 0.410 & 32.2 & 39.6 & 7.4 & 0.118 \\
\hline \multicolumn{12}{|l|}{$\begin{array}{l}\text { EORTC QLQ-BR23 } \\
\text { Functional Scale }\end{array}$} \\
\hline Body image & 205 & 62.1 & 2.0 & 63.7 & 79.6 & 15.9 & $<0.001$ & 77.7 & 53.6 & -24.0 & $<0.001$ \\
\hline Sexual functioning & 181 & 24.7 & 1.8 & 25.3 & 21.8 & -3.4 & 0.241 & 26.1 & 20.8 & -5.3 & 0.212 \\
\hline Sexual enjoyment & 68 & 41.2 & 3.3 & 42.2 & 37.5 & -4.7 & NA & 50.0 & 33.3 & -16.7 & NA \\
\hline Future perspective & 205 & 42.0 & 2.0 & 43.2 & 37.4 & -5.8 & 0.015 & 44.8 & 35.4 & 9.4 & 0.193 \\
\hline \multicolumn{12}{|l|}{$\begin{array}{l}\text { EORTC QLQ-BR23 } \\
\text { Symptom Scale }\end{array}$} \\
\hline Systemic therapy & 206 & 28.4 & 1.5 & 16.6 & 25.1 & -8.4 & $<0.001$ & 22.1 & 30.2 & -8.1 & 0.004 \\
\hline Breast symptoms & 206 & 24.5 & 1.1 & 19.1 & 26.9 & -7.9 & 0.203 & 21.2 & 36.5 & -15.3 & 0.002 \\
\hline Arm symptoms & 206 & 25.9 & 1.3 & 21.7 & 23.6 & -1.9 & $<0.001$ & 19.4 & 29.2 & -9.8 & 0.050 \\
\hline Upset by hair loss & 64 & 55.2 & 4.9 & 19.5 & 53.3 & -33.8 & 0.005 & 20.5 & 61.9 & -41.4 & 0.196 \\
\hline
\end{tabular}

HRQOL, health-related quality of life; QOL, quality of life; SD, standard deviation; Base, Baseline; 1 yr, 1-year post-diagnosis; Diff., difference; NA, not available (due to small number of participant). a) p-value was obtained from Wilcoxon signed-rank test to compare HRQOL scores measured at baseline with QOL scores measured at 1-year post-diagnosis, separately for survivors and deaths.

scales. A large difference in the QOL score between survivors and deaths was observed in several scales, including role (13.9, $\mathrm{p}=0.004)$, fatigue (13.6, $\mathrm{p}=0.001)$, dyspnea $(12.4, \mathrm{p}=0.022)$, and financial difficulties $(13.8, \mathrm{p}=0.007)$ (data not shown).

\section{Survival analysis}

Univariate and multivariate Cox regression analyses for each QOL variable are displayed in Table 3. HR in all models corresponded to a 10-point increment for HRQOL scores. From the adjusted model, higher scores in the physical (adjusted HR [aHR], 0.70; $\mathrm{p}=0.029)$, role $(\mathrm{aHR}, 0.68 ; \mathrm{p}=0.001)$, emotional ( $\mathrm{aHR}, 0.72 ; \mathrm{p}=0.012$ ) scales significantly improved survival. In contrast, higher scores in fatigue (aHR, 1.34; $\mathrm{p}=0.006)$, dyspnea (aHR, 1.29; $\mathrm{p}=0.009)$, and appetite loss $(\mathrm{aHR}, 1.24 ; \mathrm{p}=0.036)$ increased risk of death. In addition, marginally significant associations were found between global health status and future perspective scales with OS (aHR, 0.77 and 0.83 , respectively). Results were interpreted as such: for example, with the fatigue scale, a 10-point increase in fatigue score was associated with 1.34 times increased risk of death. 
QLQ-C30 functional scale-higher score indicates better OOL
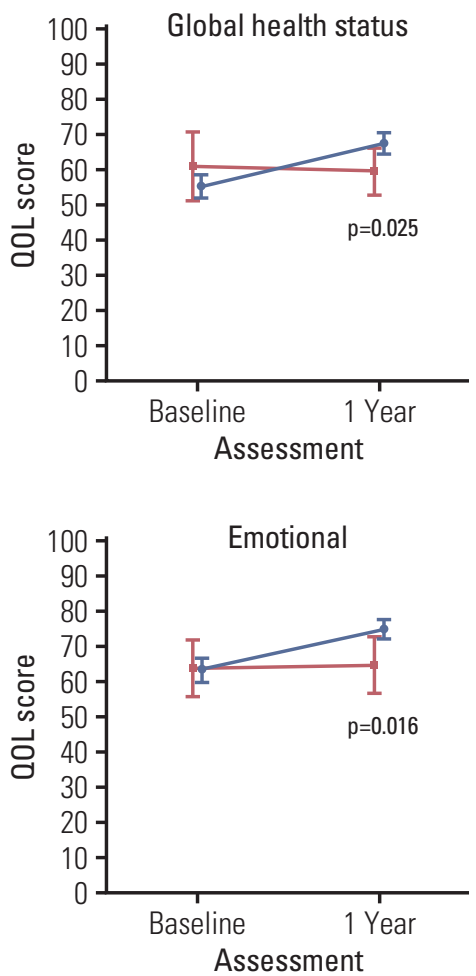
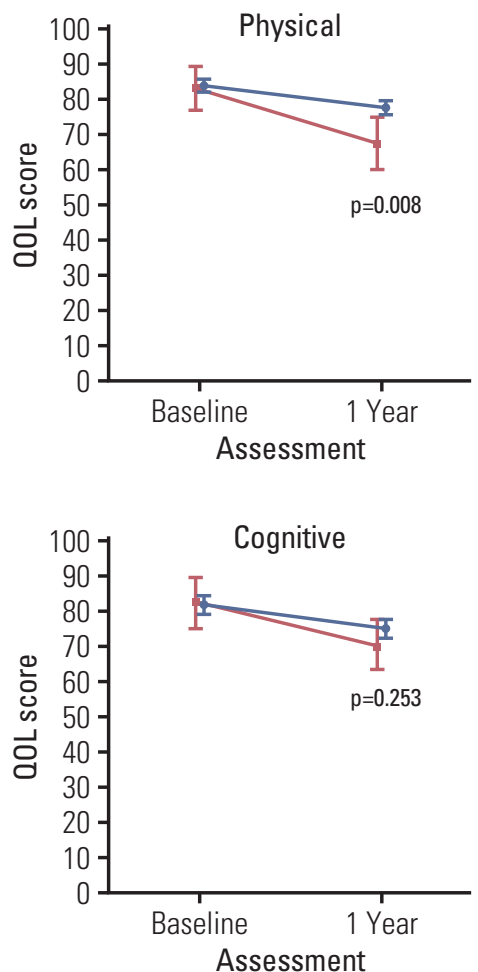
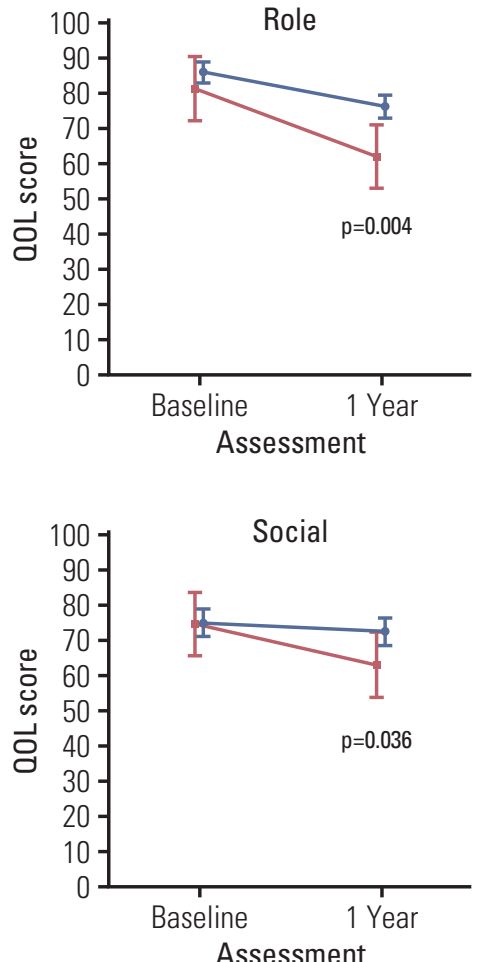

Fig. 2. Health-related quality of life (QOL) measured by QLQ-C30 functional scale after diagnosis (baseline) and at 1-year post-diagnosis (1 year). Blue color indicates the QOL mean scores and 95\% confidence intervals for survivors. Red color indicates QOL mean scores and 95\% confidence intervals for deaths. p-values were obtained using the Kruskal-Wallis test to compare QOL scores measured at 1-year post-diagnosis between survivors and deaths.

\section{Discussion}

Utilizing 1-year post-diagnosis QOL and long-term survival are two strengths exclusive to our study. While previous studies focused on correlating pretreatment QOL with survival, we shifted our interest to post-treatment QOL. In this study, the majority of our BC patients were diagnosed at stage I to III and finished their treatment as well as recovery within seven to eight months. One year after diagnosis, all participants had finished their treatment and had a few months for recovery. Given that cancer treatments have negative physical and socio-emotional consequences, assessing the patients' QOL at this time and how these QOL associated with survival are of great importance. To the best of our knowledge, this approach has not been utilized in published works and is therefore a unique aspect of this study. An additional strength of this study was the long-term followup, with the median follow-up time being more than ten years.
There are two key findings from our study. Firstly, at 1-year post-diagnosis, survivors had better HRQOL scores compared to deaths even though their health status was similar at baseline. Secondly, we found that at 1-year post-diagnosis, functional scales, including physical, role, emotional, and symptom scales, including fatigue, dyspnea were predictive for OS. HRQOL is considered one of the main study endpoints in the oncology field and provides useful prognostic information to both patients and health care providers. However, it continues to be evaluated infrequently in clinical oncology practices because of many challenges and barriers [31]. These barriers are not due to the lack of valid tools for measurement but are due to the difficulty in incorporating QOL measurements into busy clinical practices [32]. Here, there was a statistically significant relationship between several dimensions of QOL and long-term survival in BC patients. Thus, our results support the rationale to incorporate regular QOL assessment and management into oncological practices.

HRQOL at 1-year post-diagnosis of BC patients in this 
QLO-C30 symptom scale-higher score indicates worse QOL
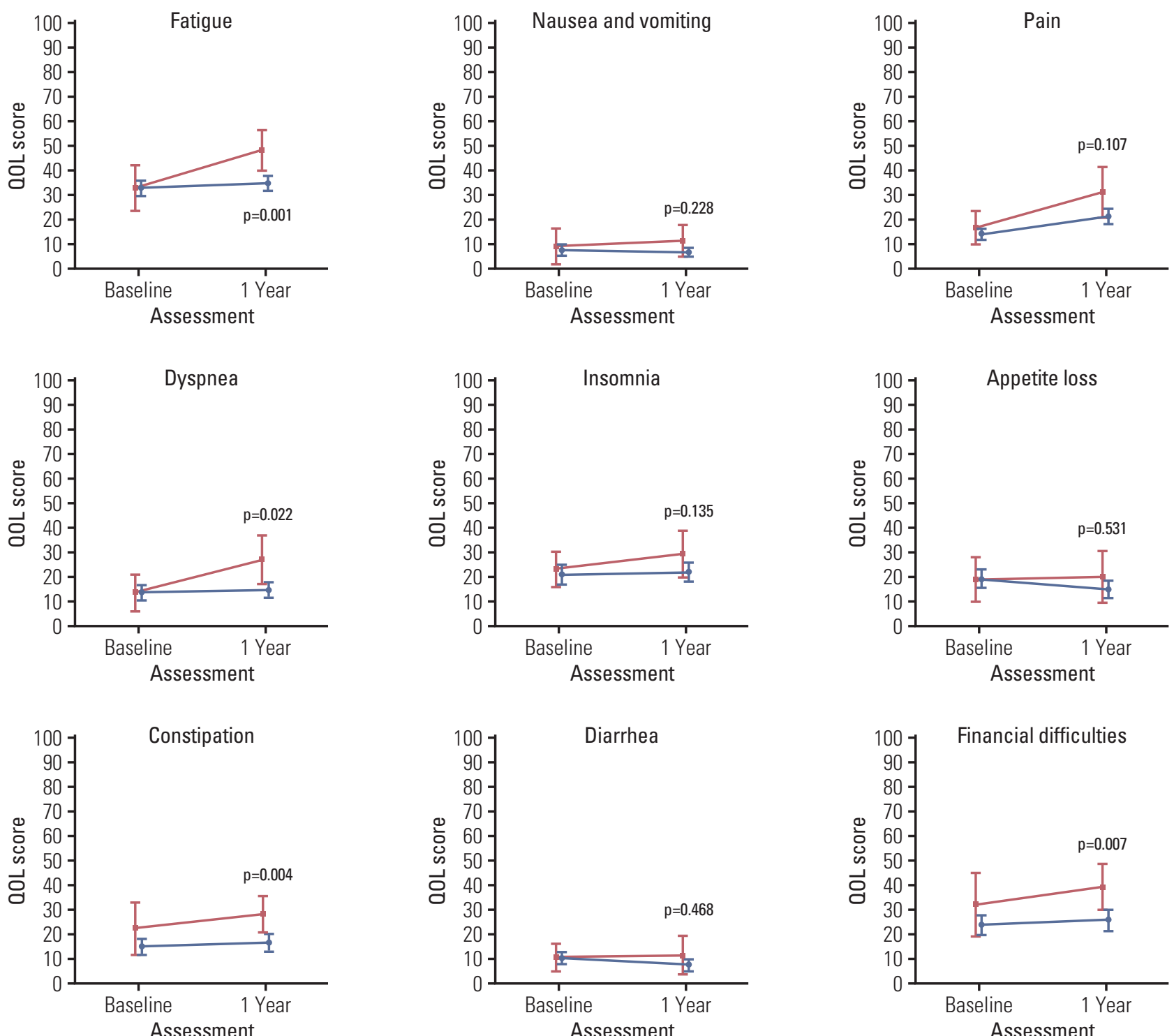

Fig. 3. Health-related quality of life (QOL) measured by QLQ-C30 symptom scale after diagnosis (baseline) and at 1-year post-diagnosis (1 year). Blue color indicates the quality of life (QOL) mean scores and 95\% confidence intervals for survivors. Red color indicates QOL mean scores and 95\% confidence intervals for deaths. p-values were obtained using the KruskalWallis test to compare QOL scores measured at 1-year post-diagnosis between survivors and deaths.

cohort was investigated in a previous publication by Lee et al. [22]. The authors evaluated the HRQOL status and compared the results with that of the general population. Their findings suggested that the improvement in HRQOL scores seen at 1 year after diagnosis was modest and did not reach the level of the general population [22]. Here, we re-evaluated the HRQOL scores at this time point but shifted our emphasis to the difference between the survivor and death groups. In QLQ-C30, the role function was measured by two questions which assessed the degree of impairment of work or daily activities and leisure-related activities. We found that the role function of patients after completing the treatment course was predictive of survival independent of disease stage, the status of the patient at baseline and other factors. Our findings for the role function scale was in line with previous studies using baseline QOL scores as predictors for survival $[5,21]$. Previous studies also found that fatigue was a significant predictive factor for survival, toge- 


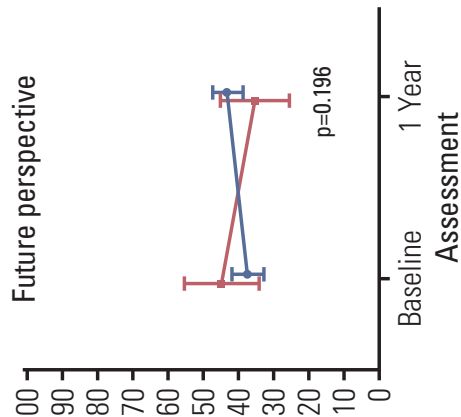

ว.0ว5 700

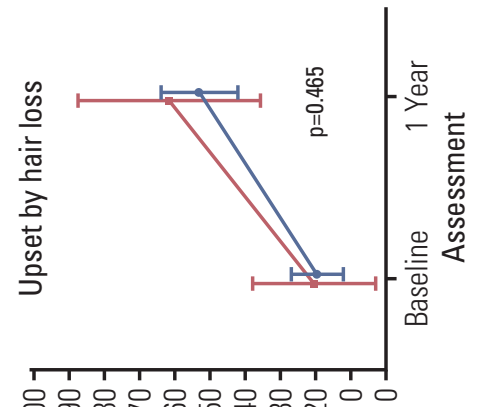

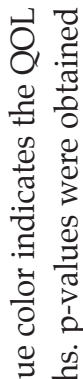

ә.0วs 700

$\stackrel{\infty}{\frac{D}{D}} \frac{\dot{D}}{\pi}$

충

구

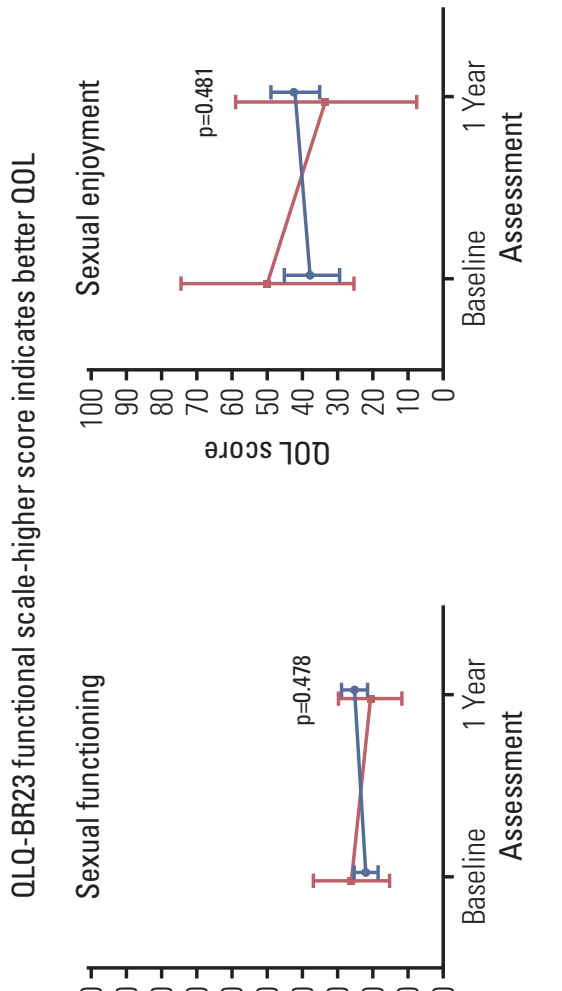

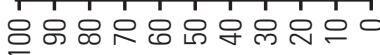

ә

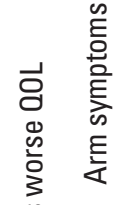

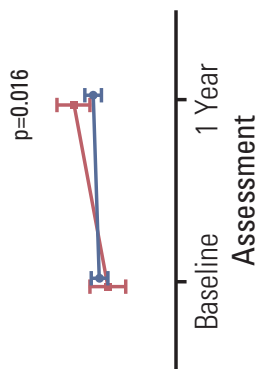

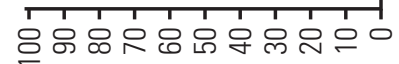

әเоэร 700

है
0
है
के
कू
ळ
के
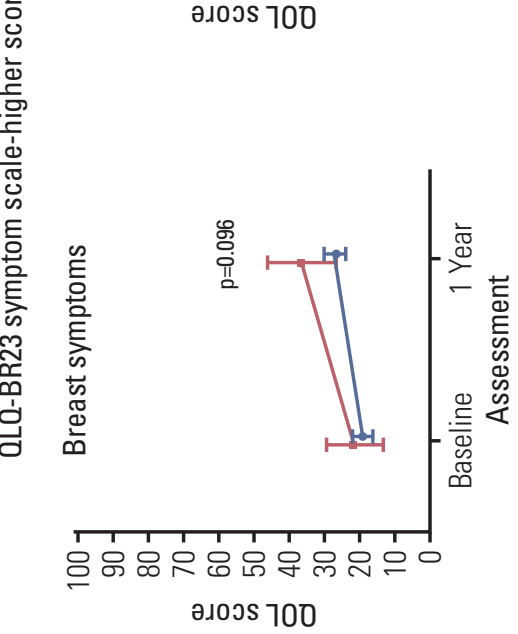

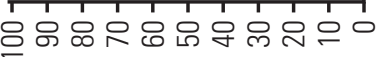

ә

$\frac{\pi}{2} e^{2}$

कृ

تิ

क :

สี ชิ

\) se

ㄴ. 느

त

(ี)

@

i

อ

क

8,

त्व

ฮัత ฮี

กิ

1)

व

ลे

Ð

อㄹำ

造

$\exists$

80

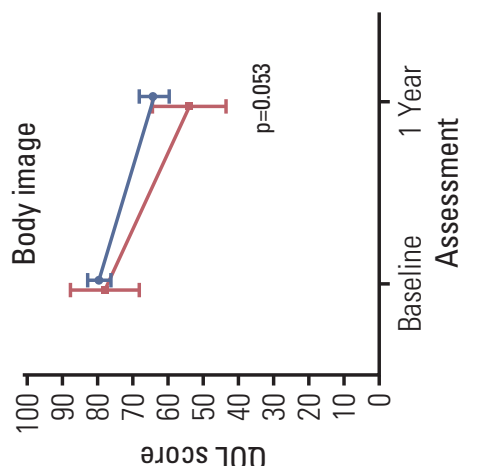

त्व
$\frac{\pi}{0}$
$\frac{0}{ \pm}$
$\frac{0}{E}$
$\frac{0}{0}$
के

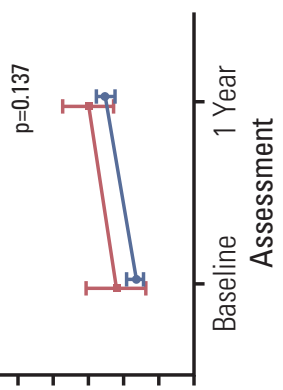

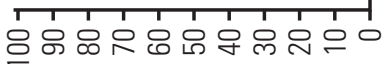

әเоวง 700

룸

줄

유

의

ป त 
Table 3. Association between HRQOL scores measured by QLQ-C30 and QLQ-BR23 at 1-year post-diagnosis and overall survival

\begin{tabular}{|c|c|c|c|c|}
\hline \multirow{2}{*}{ QOL scale } & \multicolumn{2}{|c|}{ Univariate model ${ }^{\text {a) }}$} & \multicolumn{2}{|c|}{ Adjusted model ${ }^{\text {b) }}$} \\
\hline & HR & p-value & HR & p-value \\
\hline Global health status/QOL & 0.84 & 0.037 & 0.77 & 0.047 \\
\hline \multicolumn{5}{|c|}{ EORTC QLQ-C30 Functional Scale } \\
\hline Physical & 0.71 & 0.001 & 0.70 & $0.029^{c)}$ \\
\hline Role & 0.82 & 0.002 & 0.68 & $0.001^{\mathrm{c})}$ \\
\hline Emotional & 0.82 & 0.006 & 0.72 & $0.012^{\mathrm{c})}$ \\
\hline Cognitive & 0.89 & 0.217 & 0.76 & $0.058^{c)}$ \\
\hline Social & 0.90 & 0.076 & 0.98 & $0.865^{\mathrm{d})}$ \\
\hline \multicolumn{5}{|c|}{ EORTC QLQ-C30 Symptom Scale } \\
\hline Fatigue & 1.26 & 0.001 & 1.34 & $0.006^{\mathrm{d})}$ \\
\hline Nausea and vomiting & 1.23 & 0.046 & 1.40 & $0.165^{\mathrm{d}), \mathrm{e}}$ \\
\hline Pain & 1.18 & 0.014 & 1.16 & $0.118^{\mathrm{c})}$ \\
\hline Dyspnea & 1.22 & 0.002 & 1.29 & $0.009^{c)}$ \\
\hline Insomnia & 1.09 & 0.156 & 1.15 & 0.171 \\
\hline Appetite loss & 1.09 & 0.192 & 1.24 & $0.036^{\mathrm{c}), \mathrm{d})}$ \\
\hline Constipation & 1.16 & 0.010 & 1.00 & 0.999 \\
\hline Diarrhea & 1.13 & 0.191 & 1.24 & $0.091^{\mathrm{d})}$ \\
\hline Financial difficulties & 1.14 & 0.018 & 1.03 & 0.776 \\
\hline \multicolumn{5}{|c|}{ EORTC QLQ-BR23 Functional Scale } \\
\hline Body image & 0.90 & 0.073 & 0.95 & $0.560^{c)}$ \\
\hline Sexual functioning & 0.93 & 0.449 & 0.83 & $0.182^{\mathrm{d})}$ \\
\hline Future perspective & 0.92 & 0.188 & 0.83 & 0.046 \\
\hline \multicolumn{5}{|c|}{ EORTC QLQ-BR23 Symptom Scale } \\
\hline Systemic therapy SE & 1.19 & 0.068 & 1.33 & 0.126 \\
\hline Breast symptoms & 1.12 & 0.152 & 0.98 & $0.919^{c)}$ \\
\hline Arm symptoms & 1.18 & 0.023 & 1.14 & $0.279^{c)}$ \\
\hline
\end{tabular}

HRQOL, health-related quality of life; QOL, quality of life; HR, hazard ratio; EORTC QLQ-BR23, European Organization

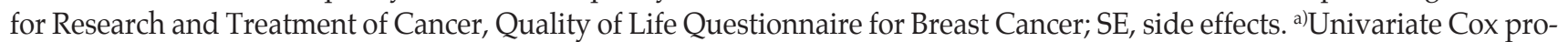
portional hazard model, include score variable of each scale, ${ }^{b}$ Multivariate Cox proportional hazard model, adjusted for age, stage at diagnosis, and baseline QOL scores. Sexual enjoyment and upset by hair lost scales were excluded due to small number of participants, ${ }^{c}$ Additional adjusted factors: duration of radiotherapy, ${ }^{d}$ Additional adjusted factors: comorbidity

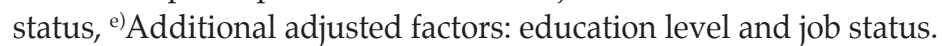

ther with pain or global QOL scale. However, HRQOL was measured at baseline, not post-treatment as in this study $[5,11,17,21]$. Our findings showed that emotional function was an independent prognostic indicator of OS. Consistent results were found in previous studies. Anxious emotion has previously been associated with poor survival in advanced cancer patients [33]. Our results add more evidences to support that interventions to improve emotional and stress management might have a positive impact on survival.

Besides the medical-related QOL scales discussed above, our findings suggest that at 1-year post-diagnosis, non-medical issues may be predictive for survival. Patients who reported more worries, depression or concerns about their future health and body image might have poorer survival, regardless of their baseline QOL status and stage of disease. Findings from this study further suggest some inequalities in financial toxicity between survivors and deaths reporting at 1-year post-diagnosis. The survivor group reported significantly less difficulties compared to the death group. However, this result did not remain statistically significant in multivariate model. In Korea, since 2004, the government has introduced a policy that decreases co-payment for cancer patients from $30 \%$ to $5 \%$ [34]. However, financial difficulties might occur even when the co-payment is not required or at low proportion [35]. Financial toxicity has been associated with not only worse survival as found in the current study and another [36], but also other factors such as lower patient satisfaction [37,38] and worse compliance [39]. For the future, 
a deeper understanding of factors causing financial difficulties among cancer patients and how these impact on QOL as well as survival is warranted.

Our study had some limitations. The first limitation is the small number of observed deaths, which was expected because we only included early-stage BC patients in our cohort. Second, we did not have information on additional factors that could affect the QOL and survival outcomes, including the duration of treatment and recovery period or the patients' access to post-treatment support systems. Furthermore, generalizability of this study was limited as the study was conducted at a single institution. Missing data on BC cancer-related scales, including sexual functioning, were also considerable. Despite these limitations, our results have important implications in both clinical and research practices. HRQOL assessments after treatment may be useful as an early sign of patient deterioration and an indication of their survival. Intervention to improve role function and symptoms, including fatigue and nausea, could have the potential to improve survival outcomes.

Our analysis provides additional prognostic information for survival beyond that available from conventional factors. Besides medical-related symptoms, non-medical aspects, functional wellbeing, and future perspective in the first year after cancer diagnosis are significant prognostic factors for
OS of BC. This suggests a possible avenue of intervention by maintaining or enhancing these functional supports for women soon after their BC diagnosis to improve disease outcomes. Regular follow-up and QOL measurements together with prompt intervention to enhance patient feelings and other associated factors might help to improve the long-term outcomes of patients.

\section{Conflicts of Interest}

Conflict of interest relevant to this article was not reported.

\section{Acknowledgments}

The authors would like to thank the BC patients who agreed to participate in this cohort study and contributed to our knowledge about HRQOL of BC patients.

This work was supported by the National Cancer Center of Korea (grant number NCC-04101502, NCC-810140-1, NCC-1710300-2, and NCC-1710300-3); TTXM was supported by the International Cooperation \& Education Program (grant number NCCRI·NCCI 5221052211, 2018). Role of the Funding Source: The funder of the study had no role in study design, data collection, data analysis, data interpretation, or writing of the report.

\section{References}

1. Gotay CC, Kawamoto CT, Bottomley A, Efficace F. The prognostic significance of patient-reported outcomes in cancer clinical trials. J Clin Oncol. 2008;26:1355-63.

2. Montazeri A. Health-related quality of life in breast cancer patients: a bibliographic review of the literature from 1974 to 2007. J Exp Clin Cancer Res. 2008;27:32.

3. Hahn EA, Cella D, Chassany O, Fairclough DL, Wong GY, Hays RD, et al. Precision of health-related quality-of-life data compared with other clinical measures. Mayo Clin Proc. 2007;82:1244-54.

4. Sprangers MA. Disregarding clinical trial-based patientreported outcomes is unwarranted: five advances to substantiate the scientific stringency of quality-of-life measurement. Acta Oncol. 2010;49:155-63.

5. Staren ED, Gupta D, Braun DP. The prognostic role of quality of life assessment in breast cancer. Breast J. 2011;17:571-8.

6. Fiteni F, Vernerey D, Bonnetain F, Vaylet F, Sennelart H, Tredaniel J, et al. Prognostic value of health-related quality of life for overall survival in elderly non-small-cell lung cancer patients. Eur J Cancer. 2016;52:120-8.

7. Montazeri A, Gillis CR, McEwen J. Measuring quality of life in oncology: is it worthwhile? II. Experiences from the treat- ment of cancer. Eur J Cancer Care (Engl). 1996;5:168-75.

8. Montazeri A, Gillis CR, McEwen J. Measuring quality of life in oncology: is it worthwhile? I. Meaning, purposes and controversies. Eur J Cancer Care (Engl). 1996;5:159-67.

9. Smyth EN, Shen W, Bowman L, Peterson P, John W, Melemed A, et al. Patient-reported pain and other quality of life domains as prognostic factors for survival in a phase III clinical trial of patients with advanced breast cancer. Health Qual Life Outcomes. 2016;14:52.

10. Gupta D, Granick J, Grutsch JF, Lis CG. The prognostic association of health-related quality of life scores with survival in breast cancer. Support Care Cancer. 2007;15:387-93.

11. Luoma ML, Hakamies-Blomqvist L, Sjostrom J, Pluzanska A, Ottoson S, Mouridsen H, et al. Prognostic value of quality of life scores for time to progression (TTP) and overall survival time (OS) in advanced breast cancer. Eur J Cancer. 2003;39: 1370-6.

12. Coates A, Gebski V, Bishop JF, Jeal PN, Woods RL, Snyder R, et al. Improving the quality of life during chemotherapy for advanced breast cancer: a comparison of intermittent and continuous treatment strategies. N Engl J Med. 1987;317:1490-5.

13. Coates A, Gebski V, Signorini D, Murray P, McNeil D, Byrne 
M, et al. Prognostic value of quality-of-life scores during chemotherapy for advanced breast cancer. Australian New Zealand Breast Cancer Trials Group. J Clin Oncol. 1992;10: 1833-8.

14. Coates AS, Hurny C, Peterson HF, Bernhard J, CastiglioneGertsch M, Gelber RD, et al. Quality-of-life scores predict outcome in metastatic but not early breast cancer. International Breast Cancer Study Group. J Clin Oncol. 2000;18:3768-74.

15. Dancey J, Zee B, Osoba D, Whitehead M, Lu F, Kaizer L, et al. Quality of life scores: an independent prognostic variable in a general population of cancer patients receiving chemotherapy. The National Cancer Institute of Canada Clinical Trials Group. Qual Life Res. 1997;6:151-8.

16. Efficace F, Therasse P, Piccart MJ, Coens C, van Steen K, Welnicka-Jaskiewicz $\mathrm{M}$, et al. Health-related quality of life parameters as prognostic factors in a nonmetastatic breast cancer population: an international multicenter study. J Clin Oncol. 2004;22:3381-8.

17. Kramer JA, Curran D, Piccart M, de Haes JC, Bruning P, Klijn $\mathrm{J}$, et al. Identification and interpretation of clinical and quality of life prognostic factors for survival and response to treatment in first-line chemotherapy in advanced breast cancer. Eur J Cancer. 2000;36:1498-506.

18. Groenvold M, Petersen MA, Idler E, Bjorner JB, Fayers PM, Mouridsen HT. Psychological distress and fatigue predicted recurrence and survival in primary breast cancer patients. Breast Cancer Res Treat. 2007;105:209-19.

19. Lehto US, Ojanen M, Dyba T, Aromaa A, Kellokumpu-Lehtinen P. Baseline psychosocial predictors of survival in localised breast cancer. Br J Cancer. 2006;94:1245-52.

20. Quinten C, Coens C, Mauer M, Comte S, Sprangers MA, Cleeland $\mathrm{C}$, et al. Baseline quality of life as a prognostic indicator of survival: a meta-analysis of individual patient data from EORTC clinical trials. Lancet Oncol. 2009;10:865-71.

21. Efficace F, Biganzoli L, Piccart M, Coens C, Van Steen K, Cufer $\mathrm{T}$, et al. Baseline health-related quality-of-life data as prognostic factors in a phase III multicentre study of women with metastatic breast cancer. Eur J Cancer. 2004;40:1021-30.

22. Lee ES, Lee MK, Kim SH, Ro JS, Kang HS, Kim SW, et al. Health-related quality of life in survivors with breast cancer 1 year after diagnosis compared with the general population: a prospective cohort study. Ann Surg. 2011;253:101-8.

23. Lee MK, Park S, Lee ES, Ro J, Kang HS, Shin KH, et al. Social support and depressive mood 1 year after diagnosis of breast cancer compared with the general female population: a prospective cohort study. Support Care Cancer. 2011;19:137992.

24. Aaronson NK, Ahmedzai S, Bergman B, Bullinger M, Cull A, Duez NJ, et al. The European Organization for Research and Treatment of Cancer QLQ-C30: a quality-of-life instrument for use in international clinical trials in oncology. J Natl Cancer Inst. 1993;85:365-76.

25. Sprangers MA, Groenvold M, Arraras JI, Franklin J, te Velde A, Muller M, et al. The European Organization for Research and Treatment of Cancer breast cancer-specific quality-of-life questionnaire module: first results from a three-country field study. J Clin Oncol. 1996;14:2756-68.

26. Lemieux J, Goodwin PJ, Bordeleau LJ, Lauzier S, Theberge V. Quality-of-life measurement in randomized clinical trials in breast cancer: an updated systematic review (2001-2009). J Natl Cancer Inst. 2011;103:178-231.

27. Yun YH, Park YS, Lee ES, Bang SM, Heo DS, Park SY, et al. Validation of the Korean version of the EORTC QLQ-C30. Qual Life Res. 2004;13:863-8.

28. Yun YH, Bae SH, Kang IO, Shin KH, Lee R, Kwon SI, et al. Cross-cultural application of the Korean version of the European Organization for Research and Treatment of Cancer (EORTC) Breast-Cancer-Specific Quality of Life Questionnaire (EORTC QLQ-BR23). Support Care Cancer. 2004;12:441-5.

29. Fayers P, Artonson NK, Bjordal K, Groenvold M, Curran D, Bottomley A. The EORTC QLQ-C30 scoring manual. 3rd ed. Brussels: European Organisation for Research and Treatment of Cancer; 2001.

30. Osoba D. Interpreting the meaningfulness of changes in health-related quality of life scores: lessons from studies in adults. Int J Cancer Suppl. 1999;12:132-7.

31. Gralla RJ, Griesinger F. Interpreting clinical trials in lung cancer: impact of methodology and endpoints. J Thorac Oncol. 2007;2 Suppl 2:S51-8.

32. Gupta D, Braun DP, Staren ED. Association between changes in quality of life scores and survival in non-small cell lung cancer patients. Eur J Cancer Care (Engl). 2012;21:614-22.

33. Zhao W, Wu Z, Chen J, Jia H, Huang Z, Chen M, et al. Survival prediction of anxious emotion in advanced cancer patients receiving palliative care. Psychooncology. 2017;26:1463-9.

34. Kim S, Kwon S. The effect of extension of benefit coverage for cancer patients on health care utilization across different income groups in South Korea. Int J Health Care Finance Econ. 2014;14:161-77.

35. Perrone F, Jommi C, Di Maio M, Gimigliano A, Gridelli C, Pignata $S$, et al. The association of financial difficulties with clinical outcomes in cancer patients: secondary analysis of 16 academic prospective clinical trials conducted in Italy. Ann Oncol. 2016;27:2224-9.

36. Ramsey SD, Bansal A, Fedorenko CR, Blough DK, Overstreet KA, Shankaran V, et al. Financial insolvency as a risk factor for early mortality among patients with cancer. J Clin Oncol. 2016;34:980-6.

37. Chino F, Peppercorn J, Taylor DH Jr, Lu Y, Samsa G, Abernethy $\mathrm{AP}$, et al. Self-reported financial burden and satisfaction with care among patients with cancer. Oncologist. 2014;19:41420.

38. Kang D, Cho J, Kim IR, Kim MK, Kim WS, Kim SJ. Healthrelated quality of life in non-hodgkin lymphoma survivors: a prospective cohort study. Cancer Res Treat. 2018;50:1051-63.

39. Zafar SY, Peppercorn JM, Schrag D, Taylor DH, Goetzinger AM, Zhong X, et al. The financial toxicity of cancer treatment: a pilot study assessing out-of-pocket expenses and the insured cancer patient's experience. Oncologist. 2013;18:381-90. 